\title{
FINITE-DIFFERENCE MODELLING OF GROUND-PENETRATING RADAR DATA: ANTENNA RADIATION PATTERNS
}

\author{
Klaus Holliger and Tim Bergmann \\ Institute of Geophysics, ETH-Hönggerberg, $\mathrm{CH}-8093$ Zürich, Switzerland
}

\begin{abstract}
SUMMARY
We present a FDTD algorithm to model the near-field radiation characteristics of dipole antennas. The antenna is represented as a wire with a small gap in the middle. The initial condition is given by a time-variable voltage pulse fed into the central gap in the wire. The resulting $\mathbf{E}$-field in the gap can be calculated analytically, whereas the $\mathbf{E}$ - and $\mathbf{H}$-fields in the vicinity of the wire are evaluated by numerical solution of an integral representation of Maxwell's equations. Integration contours and surfaces are defined by the cells surrounding the wire. Everywhere else $\mathbf{E}$ - and $\mathbf{H}$-fields are calculated using conventional FDTD approximations of Maxwell's equations. Once the source voltage pulse has decayed the FDTD approximation of Maxwell's equations is also used for the cells surrounding the wire.
\end{abstract}

\section{INTRODUCTION}

There are many similarities and analogies between seismic reflection and ground-penetrating radar (GPR) data. Acquisition, processing, and interpretation strategies used in both methods are nearly identical. One major difference is, however, that the near-field region, i.e. the part of the wavefield that is heavily affected by the source, is much more important for GPR than for seismic reflection data. All common processing and imaging techniques used in reflection seismics and adopted in GPR are based on far-field approximations. However, the far-field assumption is not valid for GPR data, in which the near-field extends to some ten wavelengths and thus comprises the better part of a typical data set. This points to the importance of a better understanding of the near-field in GPR and its effects on the data in general and on GPR data processing and interpretation in particular. Typical GPR sources and receivers consist of dipole antennas. Modelling the radiation characteristics of such antennas is commonly based on analytical methods that only consider the far-field of radiation pattern and make idealised assumptions on materials surrounding the antenna (Arcone, 1995). While such approximations are useful to account for the general directivity effects of GPR sources, they are not suitable for understanding the detailed physics in the near-field of GPR data under the complex and laterally variable conditions prevailing along the earth's surface.

To account for the variability of the air-ground interface encountered along a typical GPR survey, flexible numerical solutions are required. Carcione (1996) incorporated antenna radiation patterns in finite-difference solutions of Maxwell's equations by superposing various anisotropic point sources to emulate an analytical far-field response. Although this approach solves the non-trivial task of including radiation patterns into grid methods, it offers no more physical insight. To overcome this problem, we present a FDTD approximation of Maxwell's equations that realistically models the detailed near-field characteristics of GPR data. 


\section{FDTD ANALYSIS OF ELECTROMAGNETIC FIELDS IN THE VICINITY OF THIN WIRES}

Dipole antennas used in GPR surveys essentially consist of a wire with a narrow gap in the middle. Therefore, the following analysis is based on a FDTD technique originally developed in electrical engineering for estimating electromagnetic fields in the vicinity of thin wires and plates (Taflove et al., 1988). Figure 1 depicts a thin wire running parallel to the $y$-axis of a staggered finite-difference grid (Yee, 1966). Also shown are the electromagnetic field components on the grid points surrounding this wire.
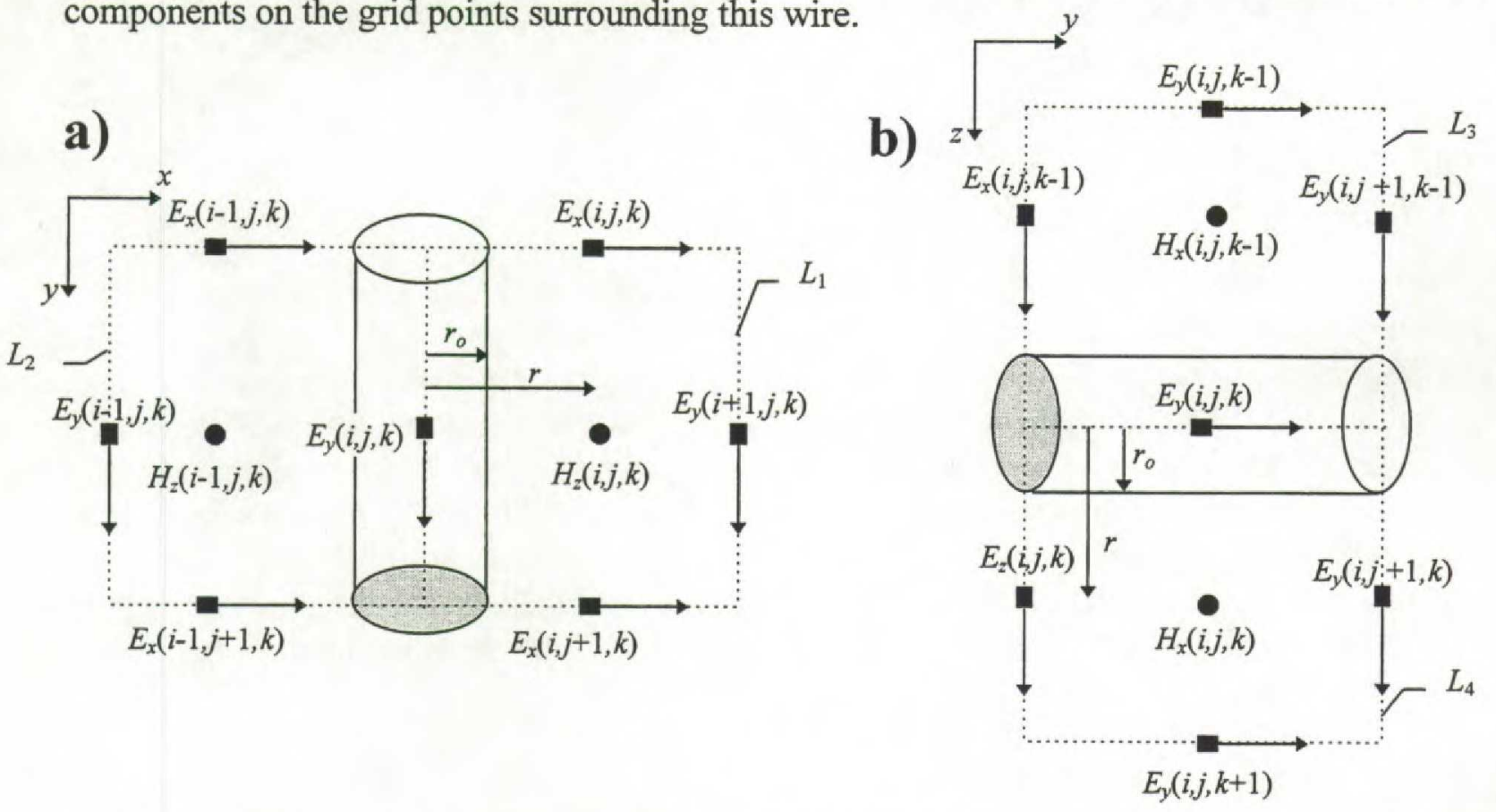

Figure 1. 2-dimensional projections of a thin wire running parallel to the $y$-axis of a 3dimensional staggered-finite difference grid. a) $x y$-plane, b) $y z$-plane.

Provided that the radius $r_{o}$ of the wire is much smaller than the mesh width of the finite-difference grid and the dominant wavelength, the $\mathbf{E}$ - and $\mathbf{H}$-field components in the immediate vicinity of the wire will decay as $1 / r$. We thus obtain:

$$
\begin{aligned}
& H_{x}(i, j, r) \approx H_{x}(i, j, k) \frac{\Delta z}{2} \frac{1}{r} \\
& H_{z}(r, j, k) \approx H_{z}(i, j, k) \frac{\Delta x}{2} \frac{1}{r} \\
& E_{x}(r, j, k) \approx E_{x}(i, j, k) \frac{\Delta x}{2} \frac{1}{r} \\
& E_{z}(i, j, r) \approx E_{z}(i, j, k) \frac{\Delta z}{2} \frac{1}{r}
\end{aligned}
$$

with $r$ being the radial distance from the centre of the wire and the indices $(i, j, k)$ referring to the finite-difference coordinates of the $E_{y}$-component in the centre of the wire (Figure 1). Let us now consider an integral form of Maxwell's equations, also known as Faraday's law:

$$
\oint_{L} \mathbf{E} \cdot \mathbf{d l}=-\mu \frac{\partial}{\partial t} \int_{S} \mathbf{H} \cdot \mathbf{d s},
$$


where $L$ is the integration contour and $S$ the surface bounded by it. By integrating along the cell boundaries of the $\mathbf{E}$-field to the right of the wire axis in Figure $1 \mathrm{a}\left(L_{1}\right)$ and assuming that $E_{y}$ is uniform within one grid cell we thus obtain:

$$
\begin{aligned}
& -E_{y}(i, j, k)+\int_{r_{0}}^{\Delta x} E_{x}(i, j, k) \frac{\Delta x}{2} \frac{d r}{r}+E_{y}(i+1, j, k) \Delta y-\int_{r_{\theta}}^{\Delta x} E_{x}(i, j+1, k) \frac{\Delta x}{2} \frac{d r}{r} \\
& =-\mu(i, j, k) \frac{\partial}{\partial t} \int_{r_{0}}^{\Delta x} H_{z}(i, j, k) \frac{\Delta x \Delta y}{2} \frac{d r}{r}
\end{aligned}
$$

Solving the integrals and bearing in mind that $E_{y}=0$ along the wire axis yields:

$$
\begin{aligned}
& 0+E_{x}(i, j, k) \frac{\Delta x}{2} \ln \left(\frac{\Delta x}{r_{o}}\right)+E_{y}(i+1, j, k) \Delta y-E_{x}(i, j+1, k) \frac{\Delta x}{2} \log \left(\frac{\Delta x}{r_{o}}\right) \\
& =-\mu(i, j, k) \frac{\Delta x \Delta y}{2} \ln \left(\frac{\Delta x}{r_{o}}\right) \frac{\partial H_{z}(i, j, k)}{\partial t}
\end{aligned}
$$

Finally, approximating the continuous time derivative with a discrete difference operator and rearranging results the finite-difference equation:

$$
\begin{gathered}
H_{z}^{n+1 / 2}(i, j, k)=H_{z}^{n-1 / 2}(i, j, k)+\frac{\Delta t}{\mu(i, j, k) \Delta y}\left(E_{x}^{n}(i, j+1, k)-E_{x}^{n}(i, j, k)\right) \\
-\frac{2 \Delta t}{\mu(i, j, k) \Delta x \ln \left(\frac{\Delta x}{r_{o}}\right)} E_{y}^{n}(i+1, j, k)
\end{gathered}
$$

Similarly, by integrating along $L_{2}, L_{3}$, and $L_{4}$, respectively, we obtain for the remaining three $\mathrm{H}$-field components in the immediate vicinity of the wire:

$$
\begin{aligned}
H_{z}^{n+1 / 2}(i-1, j, k) & =H_{z}^{n-1 / 2}(i-1, j, k)+\frac{\Delta t}{\mu(i-1, j, k) \Delta y}\left(E_{x}^{n}(i-1, j+1, k)-E_{x}^{n}(i-1, j, k)\right) \\
& +\frac{2 \Delta t}{\mu(i-1, j, k) \Delta x \ln \left(\frac{\Delta x}{r_{o}}\right)} E_{y}^{n}(i-1, j, k) \\
H_{x}^{n+1 / 2}(i, j, k-1)= & H_{x}^{n-1 / 2}(i, j, k-1)+\frac{\Delta t}{\mu(i, j, k-1) \Delta y}\left(E_{z}^{n}(i, j, k-1)-E_{z}^{n}(i, j+1, k)\right) \\
& -\frac{2 \Delta t}{\mu(i, j, k-1) \Delta z \ln \left(\frac{\Delta z}{r_{o}}\right)} E_{y}^{n}(i, j, k-1)
\end{aligned}
$$




$$
\begin{aligned}
H_{x}^{n+1 / 2}(i, j, k) & =H_{x}^{n-1 / 2}(i, j, k)+\frac{\Delta t}{\mu(i, j, k) \Delta y}\left(E_{z}^{n}(i, j, k)-E_{z}^{n}(i, j+1, k)\right) \\
& +\frac{2 \Delta t}{\mu(i, j, k) \Delta z \ln \left(\frac{\Delta z}{r_{o}}\right)} E_{y}^{n}(i, j, k+1)
\end{aligned}
$$

\section{FDTD Algorithm for Modelling Dipole ANTEnNa Radiation Patterns}

In conjunction with a suitable initial condition and a standard staggered-grid FDTD approximation of Maxwell's equations (Yee, 1966; Bergmann et al., 1996), equations 5 through 8 thus allow us to calculate the electromagnetic field in the vicinity of wire surrounded by materials with arbitrary and laterally variable constitutive parameters $(\varepsilon, \mu, \sigma)$. For the calculation of the radiation of dipole antennas used in GPR we consider a wire with a narrow gap in the middle. The width of the gap is $n \Delta y$ with $n=1,2,3, \ldots$. In the case of an emitting antenna, this gap is fed by a time-variant voltage pulse $V(t)$, which results in the following E-field within the gap: $\mathbf{E}(t)=\left(0, E_{y}(t)=V(t) / n \Delta y, 0\right)$, whereas $E_{y}(t)=0$ everywhere else along the wire. This is the initial condition required to start the FDTD calculation, which then proceeds according to the following algorithm:

1. Update the standard FDTD equations using the above initial condition.

2. Evaluate the $\mathbf{H}$-field components in the immediate vicinity of the wire using equations 5 through 8 and overwrite the corresponding field components calculated in step 1.

3. Repeat steps 1 and 2 until the source voltage pulse $V(t)$ has decayed below a certain threshold.

4. Continue to update the electromagnetic field throughout the entire grid using the standard FDTD equations.

\section{Conclusions}

We have presented a FDTD algorithm to explicitly evaluate the near-field of GPR data originating from pulse dipole antennas surrounded by arbitrary materials. With minor modifications the same algorithm can also be used to estimate the effects of the receiving dipole antenna on reflected GPR data. The algorithm is only marginally more expensive than a standard 3-dimenisonal FDTD algorithm and can be obtained by relatively minor modification of the source insertion procedure of such an standard algorithm.

\section{REFERENCES}

Arcone, S.A., 1995, Numerical studies of radiation patterns of resistively loaded dipoles: J. Appl. Geophys., 33, 39-52.

Bergmann, T., Robertsson, J.O.A., and Holliger, K., 1996, Numerical properties of staggered finite-difference solutions of Maxwell's equations for ground-penetrating radar modeling: Geophys. Res. Lett., 23, 45-48.

Carcione, J.M., 1996. Radiation patterns for GPR forward modeling: Exp. Abst. 66th Ann. Meet. Soc. Expl. Geophys., Denver, Colorado, pp. 805-808.

Taflove, A., Umashankar, K.R., Beker, B., Harfoush, F., and Yee, K.S., 1988, Detailed FDTD analysis of electromagnetic fields penetrating narrow slots and lapped joints in thick conducting screens: IEEE Trans. Ant. Prop., 36, 247-257.

Yee, K.S., 1966, Numerical solution of initial boundary value problems involving Maxwell's equations in isotropic media: IEEE Trans. Ant. Prop., 14, 302-307. 